\title{
FONCTION DE HILBERT-SAMUEL DANS LES ANNEAUX LOCAUX RÉGULIERS NON-COMMUTATIFS
}

\author{
par J. ALEV \\ (Received 7 July, 1981)
}

Introduction. En algèbre non-commutative, on dit qu'un anneau noethérien $A$ est local si:

(i) le radical de Jacobson $M$ de $A$ est un idéal maximal,

(ii) $\cap M^{n}=(0)$,

(iii) $A / M$ est artinien simple.

Dans [9], Walker définit un anneau local régulier comme un anneau local $A$ dont le radical de Jacobson $M$ est engendré par une $A$-suite centralisante $x_{1}, x_{2}, \ldots, x_{t}$ [4], et démontre alors que:

$$
t=\operatorname{cldim} A=\operatorname{Kdim} A=\operatorname{rgldim} A=\mathrm{pd}_{A} A / M .
$$

Plus tard, dans [7], Smith donne deux classes d'exemples pour de tels anneaux:

(1) Soit $\mathscr{G}$ une algèbre de Lie nilpotente de dimension finie sur un corps $K$ et $U(\mathscr{G})$ son algèbre enveloppante. Si $\mathscr{P} \in \operatorname{Spec} U(\mathscr{G}), U(\mathscr{G})_{\mathscr{P}}$ est un anneau local régulier.

(2) Soit $R$ un anneau commutatif super-régulier. Considérons un groupe $G$ de type fini contenant un sous-groupe normal fini $H$ avec $|H|^{-1} \in R$ et $G / H$ nilpotent sans torsion. Soit $R[G]$ l'anneau de groupe. Si $\mathscr{P} \in \operatorname{Spec} R[G], R[G]_{\mathscr{P}}$ est un anneau local régulier.

Dans cette note, nous considérons la fonction $f_{M}(\dot{n})=l_{A}\left(A / M^{n+1}\right)$, qui n'est pas polynomiale en général et nous montrons qu'elle est à croissance polynomiale de façon qu'un analogue du théorème de Hilbert-Samuel pour les anneaux locaux noethériens reste vrai. Nous nous plaçons dans les anneaux locaux réguliers, parce qu'on ne connaît pas de moyen pour construire un analogue des systèmes de paramètre dans un anneau local où tout idéal est engendré par un système centralisant.

\section{Propriété d'Artin-Rees forte et Anneau de Roseblade.}

1.1. Soit $A$ un anneau noethérien et $I$ un idéal bilatère de $A$. On dit que $I$ vérifie la propriété d'Artin-Rees forte si l'anneau gradué:

$$
A \oplus I t \oplus I^{2} t^{2} \oplus \ldots \subset A[t]
$$

est noethérien. On a alors la propriété bien connue:

(P) Soit $M$ un $A$-module (à gauche) de type fini et $N$ un sous-module de $M$. Il existe un entier $k$ tel que $I^{n} M \cap N=I^{n-k}\left(I^{k} M \cap N\right)$ pour $n \geqslant k$. On ne sait pas si un idéal engendré par un système centralisant possède la propriété d'Artin-Rees forte [3], [5], [8].

1.2. Soit $A$ un anneau et $I$ un idéal de $A$. On dit que $I$ est polycentral de hauteur $c$, s'il existe une chaîne d'idéaux

$$
\begin{aligned}
& I=I_{1} \supseteq I_{2} \supseteq \ldots \supseteq I_{c} \supseteq I_{c+1}=(0), \\
& \text { Glasgow Math. J. } 24 \text { (1983) 65-69. }
\end{aligned}
$$


telle que $I_{i} / I_{i+1}$ est engendré par des éléments centraux de $A / I_{i+1}$. Dans [6], Roseblade considère le sous-anneau $\tilde{A}\left(I ; I_{1}, I_{2}, \ldots, I_{c+1}\right)$ de $A[t]$ engendré par:

$$
A, I_{c} t, I_{c} t^{2}, \ldots, I_{c} t^{2^{c-1}}, I_{c-1} t, \ldots, I_{c-1} t^{2^{c-2}}, \ldots, I_{2} t, I_{2} t^{2}, I_{1} t
$$

et montre qu'il est noethérien. On a:

$$
\tilde{A}\left(I ; I_{1}, I_{2}, \ldots, I_{c+1}\right)=A \oplus L_{1}(I) t \oplus L_{2}(I) t^{2} \oplus \ldots
$$

ce qui permet d'attacher à l'idéal $I$ une suite décroissante d'idéaux $\left\{L_{n}(I)\right\}_{n \geqslant 1}$ telle que:

$$
L_{n}(I)=I_{r+2}+\sum_{\substack{i+j=n \\ i j>0}} L_{i}(I) L_{j}(I)
$$

si $n>0, r \geqslant-1$ et $2^{r}<n \leqslant 2^{r+1}$. De plus, $I^{n} \subset L_{n}(I)$ pour tout $n$ et $L_{n}(I) \subset I^{r}$ si $r \geqslant 0$ et $(r-1) 2^{c-1}<n \leqslant r 2^{c-1}$. Soit maintenant $M$ un $A$-module de type fini et $N$ un sous-module de $M$. La noethérianité de $\tilde{A}\left(I ; I_{1}, I_{2}, \ldots, I_{c+1}\right)$ donne:

$\left(\mathrm{P}^{\prime}\right)$ Il existe un entier $k$ tel que:

$$
L_{n}(I) M \cap N=\sum_{i=0}^{k} L_{n-i}(I)\left(L_{i}(I) M \cap N\right)
$$

si $n \geqslant k$.

La suite de cette note consiste à montrer que pour notre but il suffit d'utiliser la propriété $\left(\mathrm{P}^{\prime}\right)$ qui peut s'interpréter comme une propriété d'Artin-Rees forte de la suite $\left\{L_{n}(I)\right\}_{n \geqslant 1}$, qui définit sur $A$ la même topologie que la topologie $I$-adique.

\section{Les fonctions $f_{I}$ et $g_{I}$.}

2.1. Soit $A$ un anneau noethérien et $I$ un idéal bilatère de $A$ engendré par un système centralisant $x_{1}, x_{2}, \ldots, x_{t}$. Supposons de plus que $A / I$ est artinien. Posons: $f_{I}(n)=l_{A}\left(A / I^{n+1}\right)$ et $g_{I}(n)=l_{A}\left(A / L_{n+1}(I)\right)$, où $\left\{L_{n+1}(I)\right\}_{n \geqslant 0}$ est la suite attachée à l'idéal $I$ en utilisant l'anneau de Roseblade relativement à la suite d'idéaux $I_{j}=$ $A x_{1}+A x_{2}+\ldots+A x_{t-j+1}$ pour $1 \leqslant j \leqslant t$ et $I_{t+1}=(0)$. Il est clair que $g_{I}(n) \leqslant f_{I}(n)$, mais en fait le théorème 3.1 montre que ces deux fonctions ont la même croissance dans le sens suivant.

2.2. Définition. Soit $f$ une fonction croissante définie sur $N$ à valeurs entières positives. Nous allons supposer que:

(a) Il existe un polynôme $q(X) \in Q[X]$ tel que $f(n) \leqslant q(n)$ pour tout entier naturel $n$.

(b) Il existe un entier $l>0$ et un polynôme $p(X) \in Q[X]$ tels que: $0<p(n) \leqslant f(n)$ pour $n \neq 0, n \equiv 0(l)$.

Il est alors clair qu'en présence de la condition (a), il existe un polynôme de plus haut degré $d$ vérifiant la condition (b). En particulier, $d \leqslant \operatorname{deg} q(X)$. Cet entier $d$, uniquement déterminé par $f$ sera dit "degré de $f$ " et sera noté deg $f$.

2.3. Soit $x$ un élément central dans $A$ et $J$ un idéal bilatère de $A$. Notons par $J: A x$ l'idéal $\{b / b A x \subseteq J\}=\{b / b x \in J\}$. 
Lemme. Avec les notations du 2.1, on a:

et

$$
f_{I / A x_{1}}(n)=f_{I}(n)-l_{A}\left(A / I^{n+1}: A x_{1}\right)
$$

$$
g_{I / A x_{1}}(n)=g_{I}(n)-l_{A}\left(A / L_{n+1}(I): A x_{1}\right) .
$$

Preuve. La preuve de la première égalité est la même que celle du cas commutatif $\left[10\right.$, p. 285]. Pour la deuxième égalité remarquons qu'on a $\overline{L_{n}(I)}=L_{n}(\bar{I})$ par construction dans l'anneau $A / A x_{1}$. D'autre part,

$$
\begin{aligned}
g_{\text {I/Ax }}(n) & =l_{A}\left(A / L_{n+1}(I)+A x_{1}\right) \\
& =l_{A}\left(A / L_{n+1}(I)\right)-l_{A}\left(L_{n+1}(I)+A x_{1} / L_{n+1}(I)\right) \\
& =g_{I}(n)-l_{A}\left(A x_{1} / L_{n+1}(I) \cap A x_{1}\right) .
\end{aligned}
$$

Mais, $A x_{1} / L_{n+1}(I) \cap A x_{1}$ est isomorphe à $A / L_{n+1}(I): A x_{1}$ par la multiplication par $x_{1}$, d'où le résultat.

2.4. La proposition suivante donne la majoration commutative de la fonction $f_{I}$.

Proposition. Avec les notations du 2.1 , on a: $f_{I}(n) \leqslant\left(\begin{array}{c}n+t \\ t\end{array}\right) l_{A}(A / I)$.

Preuve. Par récurrence sur $t$. Pour plus de détails voir [1].

3.1. ThÉoRÈme. Soit $A$ un anneau noethérien et I un idéal propre de $A$ tel que $A / I$ soit artinien. Supposons que I soit engendré par une A-suite centralisante $x_{1}, x_{2}, \ldots, x_{i}$. Alors les fonctions $f_{I}$ et $g_{I}$ sont de degré $t$ au sens de la définition 2.2.

Preuve. Par récurrence sur $t$.

Si $t=1, I=A x_{1}$ et $I^{k+1} \mp I^{k}$ car $x_{1}$ est non diviseur de zéro et non inversible. Comme $A x_{1}^{k} / A x_{1}^{k+1}$ est isomorphe à $A / I$, on a: $f_{I}(n)=g_{I}(n)=(n+1) l_{A}(A / I)$.

Supposons $t>1$. Tout d'abord, la proposition 2.4 assure que la condition (a) de la definition 2.2 est vérifiée. Considérons ensuite l'anneau $\bar{A}=A / A x_{1}$ et l'idéal $\bar{I}=I / A x_{1} . \bar{A}$ et $\bar{I}$ vérifient la condition du théorème avec $t-1$. Il s'ensuit par récurrence qu'il existe un entier $l_{1}$ strictement positif et un polynôme $p(X)$ à coefficients rationnels tels que:

$$
0<p(n) \leqslant g_{I / A x_{1}}(n) \text { pour } n \neq 0, \quad n \equiv 0\left(l_{1}\right), \quad \operatorname{deg} p(X)=t-1 .
$$

D'autre part, la suite $\left\{L_{n}(I)\right\}_{n \geqslant 1}$ vérifie la propriété d'Artin-Rees forte et par conséquent il existe un entier $k_{1}$ tel que pour $n \geqslant k_{1}$ on a:

$$
L_{n}(I) \cap A x_{1}=\sum_{i=0}^{k_{1}} L_{n-i}(I)\left(L_{i}(I) \cap A x_{1}\right) \subseteq \sum_{i=0}^{k_{1}} L_{n-i}(I) x_{1} \subseteq L_{n-k_{1}}(I) x_{1},
$$

ce qui implique que $L_{n}: A x_{1} \subset L_{n-k_{1}}$ puisque $x_{1}$ est non diviseur de zéro. D'après le lemme 2.3 on peut écrire:

$$
g_{I / A x_{1}}(n) \leqslant g_{I}(n)-l_{A}\left(A / L_{n+1-k_{1}}(I)\right)
$$


et par conséquent

$$
g_{I / A x_{1}}(n) \leqslant g_{I}(n)-g_{I}\left(n-k_{1}\right) \text { pour } n \geqslant k_{1} .
$$

Posons $k=l_{1} k_{1}$. Il est clair que (3.1.1) et (3.1.2) restent vrais si $l_{1}$ et $k_{1}$ sont remplacés par k. On a donc:

$$
0<p(n) \leqslant g_{I / A x_{1}}(n) \leqslant g_{I}(n)-g_{I}(n-k)
$$

pour $n \geqslant k, n \equiv 0(k)$ et $\operatorname{deg} p(X)=t-1$. Prenons $n=u k$. On obtient alors les inégalités suivantes:

$$
\begin{aligned}
& 0<p(u k) \leqslant g_{I}(u k)-g_{I}(u k-k), \\
& 0<p(u k-k) \leqslant g_{I}(u k-k)-g_{I}(u k-2 k), \\
& \ldots \\
& 0<p(2 k) \leqslant g_{I}(2 k)-g_{I}(k), \\
& 0<p(k) \leqslant g_{I}(k) .
\end{aligned}
$$

On en déduit: $0<\sum_{i=1}^{u} p(i k) \leqslant g_{I}(u k)$. Maintenant, $\sum_{i=1}^{u} p(i k)$ est un polynôme en $u k$, à coefficients rationnels et de degré $t$. En effet, il suffit de le montrer en supposant que $p(X)$ est un monôme de degré $t-1, a X^{t-1}$. On a alors: $\sum_{i=1}^{u} a(i k)^{t-1}=a k^{t-1} \sum_{i=1}^{u} i^{t-1}$. Comme d'autre part, $\sum_{i=1}^{u} i^{t-1}$ est un polynôme en $u$ de degré $t$, à coefficients rationnels, il existe $b_{0}, b_{1}, \ldots, b_{t}$ rationnels avec $b_{\imath} \neq 0$ et tels que: $\sum_{i=1}^{u} i^{t-1}=\sum_{j=0}^{1} b_{j} u^{i}$. Il s'ensuit que:

$$
\sum_{i=1}^{u} a(i k)^{t-1}=a k^{t-1} \sum_{j=0}^{t} b_{j} u^{i}=\sum_{j=0}^{t} a b_{j} k^{t-j-1}(u k)^{j} .
$$

On obtient donc le résultat cherché pour la fonction $g_{I}$ et par conséquent pour la fonction $f_{\text {r. }}$.

3.2. Corollaire. Soit $A$ un anneau local régulier et $M$ son radical de Jacobson engendré par la A-suite centralisante $x_{1}, x_{2}, \ldots, x_{r}$. Alors le degré de la fonction $f_{M}$ est t.

3.3. Remarque. Dans la définition 2.2 , si les deux polynômes $p$ et $q$ ont le même degré comme c'est le cas dans le théorème 3.1, alors:

$$
\varlimsup_{n \rightarrow \infty} \frac{\log f(n)}{\log n}=\operatorname{deg} f
$$

En particulier, la dimension au sens de Gelfand-Kirillov de $f$ est égale à deg $f$ comme on peut le trouver dans [2].

\section{RÉFÉRENCES}

1. J. Alev, Polynôme de Hilbert-Samuel dans les algèbres enveloppantes et les algèbres de groupe, Israel J. Math. 37 (1980), 231-240. 
2. W. Bohro und H. Kraft, Über die Gelfand-Kirillov-Dimension, Math. Ann. 220 (1976), 1-24.

3. Y. Nouazé et $P$. Gabriel, Idéaux premiers de l'algèbre enveloppante d'une algèbre de Lie nilpotente, J. Algebra 6 (1967), 77-99.

4. J. C. McConnell, The intersection theorem for a class of non-commutative rings, Proc. London Math. Soc. (3) 17 (1967), 487-498.

5. P. F. Pickel, Rational cohomology of nilpotent groups and Lie algebras, Comm. Algebra 6 (1978), 409-419.

6. J. E. Roseblade, Applications of the Artin-Rees lemma to group rings, Symposia Mathematica, Vol. XVII (Academic Press, 1976), 471-478.

7. P. F. Smith, On non-commutative regular local rings, Glasgow Math. J. 17 (1976), 98-102.

8. J. T. Stafford and N. R. Wallach, The restriction of admissible modules to parabolic subalgebras, à paraître.

9. R. Walker, Local rings and normalizing sets of elements, Proc. London Math. Soc. (3) 24 (1972), 27-45.

10. O. Zariski et P. Samuel, Commutative Algebra II, (Van Nostrand, 1960).

UNIVERsité DE PARIS VI

4 Place Jussieu

75230 Paris Cedex 05, France 